\title{
Shrinkage Estimation for Functional Principal Component Scores, with Application to the Population Kinetics of Plasma Folate
}

\author{
Fang Yao ${ }^{1}$, Hans-Georg Müller ${ }^{1,2}$, \\ Andrew J. Clifford ${ }^{3}$, Steven R. Dueker ${ }^{3}$, Jennifer Follett ${ }^{3}$, Yumei Lin ${ }^{3}$, \\ Bruce A. Buchholz ${ }^{4}$ and John S. Vogel ${ }^{4}$
}

February 16, 2003

${ }^{1}$ Department of Statistics, University of California, One Shields Ave., Davis, CA 95616

${ }^{2}$ Corresponding Author, e-mail: mueller@wald.ucdavis.edu

${ }^{3}$ Department of Nutrition, University of California, One Shields Ave., Davis, CA 95616

${ }^{4}$ Center for Accelerator Mass Spectrometry, Lawrence Livermore National Laboratory, Livermore, CA 94551 


\begin{abstract}
We present the application of a nonparametric method to perform functional principal components analysis for functional curve data that consist of measurements of a random trajectory for a sample of subjects. This design typically consists of an irregular grid of time points on which repeated measurements are taken for a number of subjects. We introduce shrinkage estimates for the functional principal component scores that serve as the random effects in the model. Scatterplot smoothing methods are used to estimate the mean function and covariance surface of this model. We propose improved estimation in the neighborhood of and at the diagonal of the covariance surface, where the measurement errors are reflected. The presence of additive measurement errors motivates shrinkage estimates for the functional principal components scores. Shrinkage estimates are developed through best linear prediction and in a generalized version, aiming at minimizing one-curve-leave-out prediction error. The estimation of individual trajectories combines data obtained from that individual as well as all other individuals. We apply our methods to new data regarding the analysis of the level of ${ }^{14} \mathrm{C}$-folate in plasma as a function of time since dosing healthy adults with a small tracer dose of ${ }^{14} \mathrm{C}$-folic acid. A time transformation was incorporated to handle design irregularity concerning the time points on which the measurements were taken. The proposed methodology incorporating shrinkage and data-adaptive features is seen to be well suited for describing population kinetics of ${ }^{14} \mathrm{C}$-folate specific activity and random effects, and can also be applied to other functional data analysis problems.
\end{abstract}

Key words: Covariance, Cross-Validation, Eigenfunctions, Functional Data, Measurement Error, Pharmacokinetics, Random Effects, Repeated Measurements, Smoothing. 


\section{Introduction}

We develop a version of functional principal components (FPC) analysis that includes shrinkage of the FPC scores as a means to handle measurement errors and improve the prediction error of the representation of individual trajectories in functional principal components analysis. We apply the new methodology to analyze the kinetics of the appearance and disappearance of ${ }^{14} \mathrm{C}$-folate in plasma of healthy adults who were given a small tracer dose of ${ }^{14} \mathrm{C}$-folic acid per os. Folate is an important vitamin, folate deficiency in pregnant mothers being associated with increased risk for spina bifida and with other disorders, and its plasma kinetics is of interest in nutritional research.

Since a parametric model will only find features in the data which are already incorporated a priori in the model, parametric approaches might be not adequate if, as is the case in our application, the time courses are not well defined and do not fall into a preconceived class of functions. In such situations an exploratory analysis through nonparametric methods is advisable. There has been increasing interest in the nonparametric analysis of data that are in the form of samples of curves or trajectories ("functional data analysis", see, e.g., Ramsay and Silverman, 1997).

Smoothing methods for analyzing functional data have been applied to detect new features in growth curves (Gasser et al., 1984). Substantial work has been done to model longitudinal data nonparametrically by estimating the eigenfunctions corresponding to the covariance function of a random curve with continuous sample curves [Berkey and Kent (1983), Besse and Ramsay (1986), Castro, Lawton, and Sylvestre (1986), Rice and Silverman (1991), Silverman (1996)]. FPCA attempts to find the dominant modes of variation around an overall trend function, and is thus a key technique in functional data analysis. In previous work, the FPC scores, which are a key feature in Karhunen-Loève representation of random trajectories, were obtained as approximations to the integrals of the defining scalar products. We propose here more a more general approach through shrinkage estimation.

The main contributions of this paper are, first, the proposed shrinkage estimates of the FPC scores, which improve upon the estimates obtained by the integration method especially when the measurements are contaminated with noise, as is usually the case in practical applications. A consequence is improved prediction of individual trajectories. Second, we demonstrate with a new data set consisting of a sample of longitudinal measurements on the kinetics of plasma folate 
the usefulness of these methods for biostatistical data.

Our approach is related to that of Staniswalis and Lee (1998), who also used scatter-plot smoothing to obtain mean and covariance functions, and proposed modifications to allow for additional measurement errors. We also propose an improved estimate for the variance of these errors through improved estimation in the neighborhood of and at the diagonal of the covariance surface, by fitting local quadratic components along the direction perpendicular to the diagonal. In practice, smoothing the covariance surface guarantees a symmetric but not always nonnegative definite estimate. We implement a simple modification where we neglect the negative eigenvalues and corresponding eigenfunctions to obtain a nonnegative definite estimate of the covariance, without changing the main characteristics of the covariance estimate. We use one-curve-leave-out cross-validation for choosing auxiliary parameters such as the degree of smoothing and the model dimension, corresponding to the number of eigenfunctions to be included, similar to Rice and Silverman (1991).

In our application, the time courses of ${ }^{14} \mathrm{C}$-folate in plasma were recorded for 13 healthy adults that were administered a small oral dose of ${ }^{14} \mathrm{C}$-folic acid $(80 \mathrm{nmol}, 100 \mathrm{nCi})$. Use of labelled nutrients and drugs is common in nutritional and pharmacologic research because it provides the only realistic way of tracking their overall fate in the body in an appropriate time frame. The time courses of plasma folate of these thirteen subjects are unknown a priori; this motivates the use of nonparametric methods for exploratory analysis. The numbers and locations of the time points on which measurements are available are irregular for these 13 subjects. Measurements are very dense during the first day, and then become increasingly sparse. This motivates a preprocessing step that consists of a time transformation. We found that a square-root logarithm transformation of time (days) is appropriate.

The remainder of the paper is organized as follows: In Section 2 we present the FPCA model incorporating measurements errors. Section 3 contains a description of the estimation of the mean, covariance surface, and eigenfunctions. The proposed shrinkage estimates for the FPC scores are discussed in Section 4. A simulation study is included in Section 5 to demonstrate the performance of the proposed methods. The application to longitudinal folate data is described in Section 6 , and concluding remarks are given in section 7 . 


\section{Modelling Trajectories Through Functional Principal Compo- nents}

We model the sample curves or trajectories as independent realizations of a stochastic process $X(t)$ that has mean $\mathrm{E}[X(t)]=\mu(t)$ and covariance function $\operatorname{cov}(X(s), X(t))=G(s, t)$. We assume that there is an orthogonal expansion (in the $L^{2}$ sense) of $G$ in terms of eigenfunctions $\phi_{k}$ and non-increasing eigenvalues $\lambda_{k}$ :

$$
G(s, t)=\sum_{k} \lambda_{k} \phi_{k}(s) \phi_{k}(t), \quad t, s \in[0, T]
$$

where $[0, T]$ is the time range of the measurements. The classical FPCA model assumes that the $i$ th random curve from the population can be expressed in a model without additional measurement errors by

$$
X_{i}(t)=\mu(t)+\sum_{k} \xi_{i k} \phi_{k}(t), \quad t \in[0, T], \quad i=1, \cdots, N
$$

where the $\xi_{k}$ are uncorrelated random variables with zero mean and variances $\mathrm{E}\left[\xi_{k}^{2}\right]=\lambda_{k}$, where $\sum_{k} \lambda_{k}<\infty$. The deviation of each sample curve from the mean is thus a sum of orthogonal curves with uncorrelated random amplitudes. We shall suppose that the mean curve and the first few eigenfunctions are smooth functions.

Often it is realistic to incorporate uncorrelated measurement errors with mean zero and constant variance $\sigma^{2}$ into the model, reflecting additional variation in the measurements, compare Rice and $\mathrm{Wu}(2000)$. Let $Y_{i j}$ be the observations of the random function $X_{i}(\cdot)$ at time points $t_{i j}$, and $\epsilon_{i j}$ additional measurement errors that are assumed to be i.i.d. and independent of the random coefficients $\xi_{i k}, i=1, \cdots, N, j=1, \cdots, n_{i}$, i.e.,

$$
Y_{i j}=X_{i}\left(t_{i j}\right)+\epsilon_{i j}=\mu\left(t_{i j}\right)+\sum_{k=1}^{\infty} \xi_{i k} \phi_{k}\left(t_{i j}\right)+\epsilon_{i j}, \quad 0 \leq t_{i j} \leq T
$$

where $\mathrm{E}\left[\epsilon_{i j}\right]=0, \operatorname{var}\left(\epsilon_{i j}\right)=\sigma^{2}$. In special cases, one might assume in addition that the $\xi_{i k}, \epsilon_{i j}$ are all jointly normally distributed, but we do not make this assumption except where explicitly noted. 


\section{Estimating the Components of the Model}

\subsection{Estimation of the mean and covariance functions}

In the functional context, it is appropriate to assume that the mean function $\mu(t)$ is a smooth curve. We use local weighted polynomial smoothing (Fan and Gijbels, 1996), fitting local lines, to estimate $\mu$ based on the pooled data from all individual curves. In practice, it is often satisfactory to choose the smoothing bandwidth subjectively, but data-adaptive methods are available, for example, see Müller and Prewitt (1993) for surface smoothing and Rice and Silverman (1991) for one-curve-leave-out cross-validation. In this method, one minimizes the cross-validation score with respect to the bandwidth $b$, given by $\operatorname{CV}(b)=\sum_{i=1}^{N} \sum_{j=1}^{n_{i}}\left\{Y_{i j}-\hat{\mu}^{(-i)}\left(t_{i j} ; b\right)\right\}^{2} / N$, where $\hat{\mu}^{(-i)}$ is the estimate of $\mu$ after removing the data of the $i$ th subject, using bandwidth $b$. For issues of smoothing dependent data, compare Lin and Carroll (2000).

We apply two-dimensional scatter-plot smoothing, based on local weighted linear smoothing and fitting local planes, to the data. Note that in model $(3), \operatorname{cov}\left(Y_{i j}, Y_{i l}\right)=\operatorname{cov}\left(X\left(t_{i j}\right), X\left(t_{i l}\right)\right)+$ $\sigma^{2} \delta_{j l}$, where $\delta_{j l}$ is 1 if $j=l$ and 0 otherwise. Let $\mathrm{C}_{i}\left(t_{i j}, t_{i l}\right)=\left(Y_{i j}-\hat{\mu}\left(t_{i j}\right)\right)\left(Y_{i l}-\hat{\mu}\left(t_{i l}\right)\right)$ be the raw covariances, where $\hat{\mu}(t)$ is the estimated mean function obtained from the previous step. It is easy to see that $\mathrm{E}\left[\mathrm{C}_{i}\left(t_{i j}, t_{i l}\right)\right] \approx \operatorname{cov}\left(X\left(t_{i j}\right), X\left(t_{i l}\right)\right)+\sigma^{2} \delta_{j l}$. Therefore the diagonal of the raw covariances should be removed, i.e., only $\mathrm{C}_{i}\left(t_{i j}, t_{i l}\right), j \neq l$, should be included as predictors in the smoothing step (Staniswalis and Lee, 1998). We again use one-curve-leave-out cross-validation, minimizing $\mathrm{CV}(h)=\sum_{i=1}^{N} \sum_{j \neq l}\left\{\mathrm{C}_{i}\left(t_{i j}, t_{i l}\right)-\widehat{G}^{(-i)}\left(t_{i j}, t_{i l} ; h\right)\right\}^{2} / N$, to choose the smoothing parameter $h$ in the surface smoothing step. Here $\widehat{G}^{(-i)}(s, t ; h)$ is the smoothed covariance function obtained by removing the $i$ th individual curve, using bandwidth $h$.

We note that the estimate $\widehat{G}(s, t)$ will always be symmetric if a symmetric weight function is used in the local linear smoothers, but not necessarily nonnegative definite for finite samples, as local linear smoothers may assign negative weights in some local windows. We implement a simple modification by neglecting negative estimates of eigenvalues and the corresponding eigenfunctions in the usual expansion of the covariance function into eigenvalues/eigenfunctions. In this way, one obtains an estimate that is guaranteed to be nonnegative definite, without changing the characteristics of the covariance estimate for larger sample sizes, where estimated eigenvalues are closer to the non-negative true eigenvalues.

The variance $\sigma^{2}$ of measurement errors is of special interest for our proposed shrinkage es- 
timates. Since the covariance of $X(t)$ is maximal along the diagonal, we expect that the shape of the surface in the direction orthogonal to the diagonal can be better approximated by a local quadratic rather than a local linear fit. Indeed, we found that the standard fitting of local planes around the diagonal leads to overestimation of $\sigma^{2}$. In order to improve the estimation in the neighborhood of and at the diagonal, we fit a local quadratic component along the direction perpendicular to the diagonal, and a local linear component in the direction of the diagonal; implementation of this local smoother is achieved easily by rotating the coordinates by $45^{\circ}$.

Prior to this smoothing step, the diagonal elements of the raw covariances, that is $C_{i}\left(t_{i j}, t_{i j}\right), i=$ $1, \ldots, N, j=1, \ldots, n_{i}$, are removed. Denote the diagonal of the resulting surface estimate by $\tilde{G}(t)$. An estimate $\hat{V}(t)$ of $\left\{G(t, t)+\sigma^{2}\right\}$ is then obtained in a second local weighted linear smoothing step, applied to the scatter plot $\left(t_{i j}, C_{i}\left(t_{i j}, t_{i j}\right)\right)$ with pooled raw variances $C_{i}\left(t_{i j}, t_{i j}\right), i=1, \cdots, N$, $j=1, \cdots, n_{i}$. To mitigate against boundary effects, we cut off the two ends of the interval to get a more stable estimate, following a suggestion of Staniswalis and Lee (1998). The resulting estimate of $\sigma^{2}$, adjusted to avoid the possibility of negative estimates, is

$$
\hat{\sigma}^{2}=\frac{2}{T} \int_{T / 4}^{3 T / 4}\{\hat{V}(t)-\tilde{G}(t)\} d t,
$$

if $\hat{\sigma}^{2}>0$ and $\hat{\sigma}^{2}=0$ otherwise.

\subsection{Estimating the Eigenfunctions and Eigenvalues}

The estimates of eigenfunctions and eigenvalues correspond to the solutions $\hat{\phi}_{k}$ and $\hat{\lambda}_{k}$ of the eigenequations, $\int_{0}^{T} \widehat{G}(s, t) \hat{\phi}_{k}(s) d s=\hat{\lambda}_{k} \hat{\phi}_{k}(t)$, where $\hat{\phi}_{k}$ are subject to $\int_{0}^{T} \hat{\phi}_{k}(t)^{2} d t=1$ and $\int_{0}^{T} \hat{\phi}_{k}(t) \hat{\phi}_{m}(t) d t=0$ for $m<k$. We estimate the eigenfunctions by discretizing the smoothed covariance, as previously described in Rice and Silverman (1991) and Capra and Müller(1997). The FPC scores $\xi_{i k}=\int\left(X_{i}(t)-\mu(t)\right) \phi_{k}(t) d t$ have traditionally been estimated by numerical integration. However, the presence of additional contaminating errors, that are pervasive in practice, motivates shrinkage estimates for the FPC scores $\xi_{i k}$ that have not yet been considered before. Since the $Y_{i j}$ are only available at discrete times $t_{i j}$, the integrals in the definition of the FPC scores $\xi_{i k}$ are usually approximated by sums, substituting $Y_{i j}$ as defined in (3) for $X_{i}\left(t_{i j}\right)$.

For notational convenience, we define $Y_{i}(t)$ as a step function with jumps at midpoints between neighboring $t_{i j}$, using $Y_{i j}$ as the size of the steps, and analogous for $\epsilon_{i}(t)$. Set $\tilde{X}_{i}=Y_{i}(t)-\epsilon_{i}(t)$, and let $\tilde{\xi}_{i k}=\int_{0}^{T}\left\{\tilde{X}_{i}(t)-\mu(t)\right\} d t$ be the discrete version of the FPC scores $\xi_{i k}$. Since neither $X_{i}$ 
nor $\tilde{X}_{i}$ is available, due to the contaminating errors $\epsilon_{i}$, we instead consider the approximations $\eta_{i k}=\int_{0}^{T}\left\{Y_{i}(t)-\mu(t)\right\} \phi_{k}(t) d t$. In practice, $\mu$ and $\phi_{k}$ are also unknown and must be estimated from the data. It is common practice, using estimates $\hat{\mu}\left(t_{i j}\right)$ for $\mu\left(t_{i j}\right)$ and $\hat{\phi}_{k}\left(t_{i j}\right)$ for $\phi_{k}\left(t_{i j}\right)$, to estimate $\eta_{i k}$ by approximating sums, letting $t_{i 0}=0$,

$$
\hat{\eta}_{i k}=\int_{0}^{T}\left(Y_{i}(t)-\hat{\mu}(t)\right) \hat{\phi}_{k}(t) d t \approx \sum_{j=1}^{n_{i}}\left(Y_{i j}-\hat{\mu}\left(t_{i j}\right)\right) \hat{\phi}_{k}\left(t_{i j}\right)\left(t_{i j}-t_{i, j-1}\right) .
$$

In order to choose the number of principal component curves that provide a reasonable approximation to the infinite-dimensional process, we use the cross-validation score based on the one-curve-leave-out prediction error. Let $\hat{\mu}_{i}^{(-i)}$ and $\hat{\phi}_{k}^{(-i)}$ be the estimated mean function and eigenfunctions after removing the $i$ th individual's curve. Then we choose the number of components $K$ to be included in the model so as to minimize the cross-validation scores

$$
\mathrm{CV}(K)=\frac{1}{N} \sum_{i=1}^{N}\left\|Y_{i}-\widehat{Y}_{i}^{(-i)}\right\|^{2}
$$

where $\|\cdot\|$ here and in the following is defined by approximating the usual $L_{2}$ distance through $\left\|Y_{i}-\widehat{Y}_{i}^{(-i)}\right\|^{2}=\sum_{j=1}^{n_{i}}\left(Y_{i j}-\widehat{Y}_{i}^{(-i)}\left(t_{i j}\right)\right)^{2}\left(t_{i j}-t_{i, j-1}\right)$. Note that $\widehat{Y}_{i}^{(-i)}$ is the predicted curve

for the $i$ th subject after removing this sample curve from fitting model $(3)$, i.e., $\widehat{Y}_{i}^{(-i)}(t)=$ $\hat{\mu}^{(-i)}(t)+\sum_{k=1}^{K} \hat{\eta}_{i k}^{(-i)} \hat{\phi}_{k}^{(-i)}(t)$.

\section{Shrinkage Estimation of Functional Principal Component Scores}

We reconsider the estimation of the functional principal components (FPC) in Model (3). Setting $\tilde{\epsilon}_{i k}=\int_{0}^{T} \epsilon_{i}(t) \phi_{k}(t) d t$, we note that $\eta_{i k}=\tilde{\xi}_{i k}+\tilde{\epsilon}_{i k}$. We conclude that the best linear predictors of the approximate FPC scores $\tilde{\xi}_{i k}$ are not given by $\eta_{i k}$, but rather by

$$
\frac{\tilde{\lambda}_{k}}{\tilde{\lambda}_{k}+\operatorname{var}\left(\tilde{\epsilon}_{i k}\right)} \eta_{i k}
$$

where $\tilde{\lambda}_{k}=\operatorname{var}\left(\tilde{\xi}_{i k}\right)$. This is a shrinkage formula, moving $\eta_{i k}$ closer towards the origin, whenever $\operatorname{var}\left(\tilde{\epsilon}_{i k}\right)>0$. If we assume that $\xi_{i k}$ and $\epsilon_{i j}$, and also that the discrete versions $\tilde{\xi}_{i k}$ and $\tilde{\epsilon}_{i k}$ are independent and jointly Gaussian, the best predictors of the approximate FPC scores $\tilde{\xi}_{i k}$ are $\mathrm{E}\left[\tilde{\xi}_{i k} \mid \eta_{i k}\right]=\tilde{\lambda}_{k} \eta_{i k} /\left\{\tilde{\lambda}_{k}+\operatorname{var}\left(\tilde{\epsilon}_{i k}\right)\right\}$, i.e., predictors $(7)$. The variation of the shrinkage estimators is reduced, observing that $\operatorname{var}\left(\eta_{i k}\right)=\operatorname{var}\left(\mathrm{E}\left[\tilde{\xi}_{i k} \mid \eta_{i k}\right]\right)+\mathrm{E}\left[\operatorname{var}\left(\tilde{\xi}_{i k} \mid \eta_{i k}\right)\right]>\operatorname{var}\left(\mathrm{E}\left[\tilde{\xi}_{i k} \mid \eta_{i k}\right]\right)$, if $\mathrm{E}\left[\operatorname{var}\left(\tilde{\xi}_{i k} \mid \eta_{i k}\right)\right]>0$. 
The quantities $\tilde{\epsilon}_{i k}$ can be approximated by the sums $\sum_{j=1}^{n_{i}} \epsilon_{i j} \phi_{k}\left(t_{i j}\right)\left(t_{i j}-t_{i, j-1}\right)$. Note that $\operatorname{var}\left(\tilde{\xi}_{i k}\right) \approx \sigma^{2} \sum_{j=1}^{n_{i}} \phi_{k}^{2}\left(t_{i j}\right)\left(t_{i j}-t_{i, j-1}\right)^{2}$, and if the number and density of measurements are sufficiently large, $\operatorname{var}\left(\tilde{\epsilon}_{i k}\right)$ can be approximated by $\sigma^{2} / n_{i}$, where $n_{i}$ is the number of repeated measurements for the $i$ th subject. A reasonable estimate of the shrinkage factor (7) is therefore

$$
\hat{\xi}_{i k}=\frac{\hat{\lambda}_{k}}{\hat{\lambda}_{k}+\hat{\sigma}^{2} / n_{i}} \hat{\eta}_{i k}
$$

where $\hat{\sigma}^{2}$ is the sample variance estimate obtained from (4), $\hat{\lambda}_{k}$ is the estimated $k$ th eigenvalue and $\hat{\eta}_{i k}$ is the raw sample estimate for the $k$ th FPC score of the $i$ th individual given in (5). In what follows, we refer to (8) as Gaussian shrinkage since it provides the appropriate shrinkage factor for the Gaussian case.

Since the presence of additional measurement errors is a main motivation of shrinkage estimation, stochastic variation in estimating the variance of the measurement errors can be expected to have an impact on the shrinkage estimates. Neglecting the variation in the estimation of eigenvalues $\lambda_{k}$, we note that the shrinkage factor $\lambda /\left(\lambda+\frac{\sigma^{2}}{n_{i}}\right)$ is a convex function of $\sigma^{2}$. Assume a multiplicative error model for the behavior of the variance estimate $\hat{\sigma}^{2}, \hat{\sigma}^{2}=\varepsilon \mathrm{E}\left[\hat{\sigma}^{2}\right]$, where $\varepsilon>0$ is a r.v. with $\mathrm{E}[\varepsilon]=1$. Then the targeted shrinkage factor is $\lambda /\left(\lambda+\frac{\mathrm{E}\left[\hat{\sigma}^{2}\right]}{n_{i}}\right)$, and from Jensen's inequality, we have

$$
\mathrm{E}\left[\lambda /\left(\lambda+\frac{\hat{\sigma}^{2}}{n_{i}}\right)\right]>\lambda /\left(\lambda+\frac{\mathrm{E}\left[\hat{\sigma}^{2}\right]}{n_{i}}\right) .
$$

We see from (9) that the appropriate shrinkage factor is smaller than that given by (8), on the average, under reasonable assumptions about randomness in the error variance. This observation motivates a generalized shrinkage method, where we replace the Gaussian shrinkage factor $\lambda /(\lambda+$ $\left.\frac{\sigma^{2}}{n_{i}}\right)$ by a generalized shrinkage factor $\lambda /\left(\lambda+\frac{\rho}{n_{i}}\right)$ for an unknown $\rho$. To achieve the target $\lambda /(\lambda+$ $\left.\frac{\mathrm{E}\left[\hat{\sigma}^{2}\right]}{n_{i}}\right)$ in the random error variance, we see that $\rho$ should be greater than $\hat{\sigma}^{2}$ on the average, which means more shrinkage is needed than provided by the Gaussian shrinkage method.

A second motivation for the generalized shrinkage method is minimization of the squared prediction error over a class of linear shrinkage factors. Let $\hat{X}_{i}(\rho)$ be the prediction for the true process $X_{i}$ obtained by using the linear shrinkage estimates $\hat{\xi}_{i k}=\hat{\lambda}_{k} \hat{\eta}_{i k} /\left(\hat{\lambda}_{k}+\frac{\rho}{n_{i}}\right)$. The orthonormality of the eigenfunctions (analogous to the derivation of Parseval's equality, see Courant and Hilbert, 1953) leads to $\left\|\hat{X}_{i}(\rho)-X_{i}\right\|^{2}=\sum_{k}\left(\hat{\lambda}_{k} \hat{\eta}_{i k} /\left(\hat{\lambda}_{k}+\frac{\rho}{n_{i}}\right)-\xi_{i k}\right)^{2}$. Setting $Z(\rho)=\frac{1}{N} \sum_{i=1}^{N}\left\|\hat{X}_{i}(\rho)-X_{i}\right\|^{2}$, $f(Z)=\min _{\rho} Z(\rho)$, the best linear predictor property of Gaussian shrinkage implies that $\rho=\hat{\sigma}^{2}$ is an approximate minimizer of $\mathrm{E}[Z(\rho)]$. Due to the concavity of $f$, using Jensen's inequality, we 
find that

$$
\mathrm{E}\left[\min _{\rho} Z(\rho)\right] \leq \min _{\rho} \mathrm{E}[Z(\rho)] \approx \mathrm{E}\left[Z\left(\hat{\sigma}^{2}\right)\right]
$$

Therefore, on the average, generalized shrinkage which corresponds to minimizing $Z(\rho)$ leads an improved estimate as compared to Gaussian shrinkage. For large sample sizes $Z(\rho)$ will be closer to $\mathrm{E}[Z(\rho)]$ and therefore the relative gain of generalized over Gaussian shrinkage is predicted to be more pronounced for smaller samples by this argument. Indeed this is what we found in simulations (Section 5).

The initial form of the generalized shrinkage formula is therefore given by $\hat{\xi}_{i k}=\frac{\hat{\lambda}_{k}}{\hat{\lambda}_{k}+\rho / n_{i}} \hat{\eta}_{i k}$, where $\rho>0$ is an unspecified shrinkage parameter. We note that Gaussian shrinkage (8) is a special case with $\rho=\hat{\sigma}^{2}$. The generalized shrinkage parameter $\rho$ is chosen in such a way as to minimize the estimated prediction error, where the estimated prediction error is obtained by one-curve-leave-out cross-validation. Let $\hat{Y}_{i}^{(-i)}(\cdot, \rho)$ be the predicted curve for the $i$ th subject, using shrinkage parameter $\rho$ and removing the data of the $i$ th subject. We then minimize the cross-validated integrated prediction error with respect to $\rho \geq 0$, to obtain

$$
\hat{\rho}=\underset{\rho \geq 0}{\arg \min } \frac{1}{N} \sum_{i=1}^{N}\left\|Y_{i}-\hat{Y}_{i}^{(-i)}(\rho)\right\|^{2},
$$

leading to the generalized shrinkage formula

$$
\hat{\xi}_{i k}=\frac{\hat{\lambda}_{k}}{\hat{\lambda}_{k}+\hat{\rho} / n_{i}} \hat{\eta}_{i k} .
$$

The generalized shrinkage method leads to the best possible shrinkage estimates in this class in terms of prediction error. It thus in general will lead to improvements over Gaussian shrinkage (8) according to (10), and Gaussian shrinkage leads to improvements over the customary FPC scores $\hat{\eta}_{i k}$, which are motivated by approximating the integrals that define the FPC scores (see Section 3.2). The customary FPC scores $\hat{\eta}_{i k}$ will nearly always overestimate the FPC scores that are optimal for prediction, and Gaussian shrinkage, while improving on this situation, often will not produce enough shrinkage especially in small sample situations.

\section{Simulation Results}

To illustrate the advantage of shrinkage estimation of FPC scores compared to customary estimation without shrinkage, we devised a simulation study, using 100 i.i.d. normal and 100 i.i.d 
non-normal samples consisting of $N=10,20,50$ random trajectories. The data were generated following model (3). The simulated process had mean function $\mu(t)=t+\sin (2 \pi t), 0 \leq t \leq 1$. We construct the covariance function of the process according to (1) from two orthonormal functions $\phi_{1}(t)=-\sqrt{2} \cos (\pi t)$, and $\phi_{2}(t)=\sqrt{2} \sin (\pi t), 0 \leq t \leq 1$. For the $i$ th partially observed path in a single sample, the number of observations, $n_{i}$, was randomly chosen between 30 to 40 . For an equally spaced grid $\left\{c_{1}, \cdots, c_{n_{i}}\right\}$ on $[0,1]$ with $c_{1}=0, c_{n_{i}}=1, d=1 /\left(n_{i}-1\right)$, the $t_{i j}$ were uniform on $\left[c_{j}-d / 2, c_{j}+d / 2\right]$, for $j=2, \cdots, n_{i}-1, t_{i 1}$ uniform on $[0, d / 2]$, and $t_{i n_{i}}$ uniform on $[T-d / 2, T]$, allowing for non-equidistant "jittered" designs. We chose $\lambda_{1}=2$ and $\lambda_{2}=1$ as the eigenvalues, $\lambda_{k}=0, j \geq 3$, and $\sigma^{2}=0.25$ as the variance of the additional measurement errors $\epsilon_{i j}$ in (3), which were assumed to be normal with mean 0.

For the 100 normal samples, the FPC scores $\xi_{i k}$ were generated from $\mathcal{N}\left(0, \lambda_{k}\right)$, while $\xi_{i k}$ in the non-normal samples were generated from a mixture of two normals, $\mathcal{N}\left(\sqrt{\lambda_{k} / 2}, \lambda_{k} / 2\right)$ with probability $1 / 2$ and $\mathcal{N}\left(-\sqrt{\left(\lambda_{k} / 2\right.}, \lambda_{k} / 2\right)$ with probability $1 / 2$. We used the cross-validation procedures described earlier for selection of bandwidths, number of eigenfunctions, and generalized shrinkage parameter $\hat{\rho}(11)$.

To demonstrate the performance of the proposed shrinkage estimates, we report in Table 1 the averages of the estimates of the variances $\hat{\sigma}^{2}$ in (4) and the mean shrinkage factor estimates for $\rho=\hat{\sigma}^{2}$ in the case of Gaussian shrinkage (8), and for $\rho=\hat{\rho}$ in the case of generalized shrinkage (12), also cross-validation scores in (11) and squared prediction errors

$$
\operatorname{SPE}(\rho)=\frac{1}{N} \sum_{i=1}^{N}\left\|Y_{i}-\hat{Y}_{i}(\rho)\right\|^{2} .
$$

We find that on the average, the generalized shrinkage (12) leads to larger downsizing of the raw estimators than Gaussian shrinkage (8) in both normal and mixture distribution situations.

For sample sizes $N=10 / 20 / 50$, and underlying normal distribution, Gaussian shrinkage results in decreases in CV of about $9 \% / 8 \% / 7 \%$ and in SPE of about $7 \% / 6 \% / 6 \%$, while for generalized shrinkage the corresponding decreases are $12 \% / 10 \% / 8 \%$ and 10\%/8\%/6\%. Just looking at decreases in SPE for the mixture distribution, Gaussian shrinkage leads to decreases of $6 \% / 4 \% / 6 \%$ for $N=10 / 20 / 50$, and generalized shrinkage to corresponding decreases of $11 \% / 9 \% / 7 \%$. We draw the following conclusions: The gains are largest for small samples, and are always larger for generalized shrinkage as compared to Gaussian shrinkage. The differences in gains between the two shrinkage methods decrease as the sample size gets larger, as was predicted in the discussion following (10) in Section 4. The generalized shrinkage parameter $\hat{\rho}$ also is seen to move closer to 
${\hat{\sigma^{2}}}^{2}$ with increasing sample size. We find that the gains do not depend much on the nature of the distribution of the principal components.

Another outcome measure of interest is the average squared error for the two FPC scores, $\operatorname{ASE}\left(\xi_{k}\right)=\sum_{i=1}^{N}\left(\hat{\xi}_{i k}-\xi_{k}\right)^{2} / N, k=1,2$, also listed in Table 1 . These errors show similar behavior as the prediction errors. With regard to all measures, the gains obtained from shrinkage as compared to no shrinkage remain substantial even for large sample sizes. Among the two shrinkage methods considered, generalized shrinkage uniformly achieves additional gains which are particularly pronounced for small sample sizes.

\section{Application to Longitudinal Plasma Folate Data}

\section{1 ${ }^{14}$ C-Folate Specific Activity}

In an experiment conducted at UC Davis, repeated measurements of the fraction of labelled folate among total folate in plasma were obtained for 13 healthy adult volunteers. Measurements were labelled by time since the volunteers orally ingested a small tracer dose of ${ }^{14} \mathrm{C}$-folic acid. The fraction of ${ }^{14} \mathrm{C}$-folate among total folate in plasma (the so-called plasma ${ }^{14} \mathrm{C}$-folate specific activity) was measured in about 20 plasma specimens drawn during the first day after dosing. After the first day, the drawing of additional blood specimens became less frequent, with a total of about 50 specimens being taken during the 200 day period after dosing.

Our main interest is characterizing the dynamic behavior of plasma ${ }^{14} \mathrm{C}$-folate specific activity in healthy adults. Methods for the collection, processing, and laboratory analyses of specimens were as described in Clifford et al. (1998). The plot of the plasma ${ }^{14} \mathrm{C}$-folate specific activity in the first four subjects versus time (after dosing) at which these measurements were taken is shown in the left panel of Figure 1 and reveals some common patterns that are described in Section 6.4.

\subsection{Time Transformation to Address Sparseness}

We note that the numbers and locations of the time points at which measurements were taken are not the same for these 13 subjects. Measurements are very dense during the first day, and then become increasingly sparse. From the left panel of Figure 1, using the original time scale, it is not easy to observe the shapes of the curves. Moreover, because we use global bandwidths in the 
smoothing steps for mean function and covariance surface, the increasing time lag between the measurements for individual subjects leads to oversmoothing when choosing an overall appropriate smoothing parameter.

Our solution to this problem is preprocessing of the data with the time transformation $t^{\prime}=$

$\sqrt{\log (1+t)}$. In fact we obtain a reasonably even distribution of measurement times after this transformation. Folate measurements versus transformed time are shown in the right panel of Figure 1 for the first four subjects. The transformed time scale is more conducive for observing the shapes of the curves and for data smoothing.

\subsection{Diagnostics for Trajectories and Outlier Detection}

Initially we applied our methods to the plasma folate data of the entire sample consisting of 13 healthy adult volunteers. Whether the trajectories recorded for the subjects come from the same population, and whether there are outliers is of biological interest. To address such questions, we desire quantities that are analogous to residuals or deviances, but apply to entire trajectories rather than traditional scatterplots. As a summary measure, we consider the integrated squared residual error in the original time scale. This criterion reflects the ability of the model to predict an observed sample curve.

Let $Y_{i}(t)$ be the step function determined by $Y_{i j}$ in the original time scale for the $i$ th subject, as described in Section 3.2, $\hat{Y}_{i}(t)$ the predicted curve, and $\left[0, T^{\prime}\right]$ the original time range for all subjects. We define the integrated squared residual error for the $i$ th subject by

$$
\mathrm{RE}_{i}=\int_{0}^{T^{\prime}}\left\{Y_{i}(s)-\hat{Y}_{i}(s)\right\}^{2} d s
$$

A large value of $\mathrm{RE}_{i}, i=1, \cdots, N$, may point to an outlying sample curve that is poorly fitted by the model.

We calculated this integrated squared residual errors for all 13 subjects, and found that $\mathrm{RE}_{2}=$ 1.6 is much larger than $\mathrm{RE}_{i} \leq 0.55, i \neq 2$. This provides some evidence that this subject is poorly fitted by the proposed model, and may be an outlier. The predicted curve and observed values of subject $\# 2$ in the original time scale are shown in Figure 2. The predicted curve is clearly underestimating the observed data after about 25 days, especially in the right tail, while the predicted curves for the other subjects are fairly close to the observations. Indeed, it was found that subject \#2 had been exposed to ${ }^{14} \mathrm{C}$ earlier through participation in an unrelated medical 
trial several years prior to the present study. This fact would have disqualified this subject from participating in the present study, but had not been known. This finding attests not only to the

astonishing accuracy of the ${ }^{14} \mathrm{C}$ detection methods used in this study, but also to the usefulness of the FPC approach for data screening and outlier detection. Since subject \#2 was thus confirmed to be a clear outlier, it was not used in the subsequent analysis. Using the integrated squared residual error $\mathrm{RE}_{i}$ in the transformed time scale leads to the same conclusion.

\subsection{Mean and Covariance Functions}

The estimated mean curve obtained using local weighted linear smoothing for the remaining 12 subjects is shown in the left panel of Figure 3. The bandwidth $b=0.11$ was chosen, guided by cross-validation. The estimated mean curve reflects the overall trend of the individual curves. One notes a short slow increase (about 4 minutes in the original time scale) in the appearance of ${ }^{14} \mathrm{C}$-folate in plasma. This delay corresponds to the time needed for the dose to reach and be absorbed from the small intestine. Then the mean plasma ${ }^{14} \mathrm{C}$-folate specific activity by time since dosing exhibits a sharp initial rise to a peak at about 2 hours (in the original time frame) as the administered dose enters the blood plasma pool. Then it drops at a decreasing rate as it enters cells where the ${ }^{14} \mathrm{C}$-folate is sequestered and converted to other chemical forms of folate. Finally, the pattern changes into a smooth slow decline toward zero.

The estimated covariance surface obtained using local weighted linear smoothing after the diagonal was removed is shown in the right panel of Figure 3. The bandwidth was $h=(0.15,0.15)$, chosen by one-curve-leave-out cross-validation. We note that the last two small eigenvalues of this covariance estimate were found to be negative. To obtain a nonnegative definite covariance surface, we implemented the simple modification described in Section 3.1, omitting the components with negative eigenvalues. The covariance surface shows high variability at the beginning with a sharp increase followed by a rapid decline. The estimate $\hat{\sigma}^{2}(4)$ for the variance $\sigma^{2}$ of the measurement error was $\hat{\sigma}^{2}=0.0037$.

\subsection{Eigenfunctions}

The smooth estimates of the first three eigenfunctions are presented in the left panel of Figure 4 (in transformed time scale). The cross-validation scores level off when more than three eigenfunctions are used, suggesting that the first three principal component curves are sufficient to describe the 
modes of variability. These three principal component functions explain about $98.5 \%$ of the total variation.

The first eigenfunction indicates that a large portion of the variability between subjects is roughly in the direction of the amplitude of the mean curve, as the first eigenfunction has a similar shape as the mean function. In particular, $89.48 \%$ of the total variability is "explained" by the first eigenfunction, which indicates that this mode of variation is dominant. The second eigenfunction takes the shape of an approximate contrast between observations around 1 hour and 4.5 hours, and contributes $6.29 \%$ to the total variation. The third eigenfunctions is indicative of a contrast of observations before and after 8 hours, and contributes $2.73 \%$ to the total variation.

\subsection{Shrinkage Estimates and Predicted Trajectories}

We applied functional principal components analysis without shrinkage, with Gaussian shrinkage (8) and with generalized shrinkage (12). Since $\hat{\sigma}^{2}=0.0037$, the additional errors in model (3) are small. The number of observations for each subject is around 40. Together with the eigenvalue estimates, 0.1052, 0.0074, and 0.0032, we that find Gaussian shrinkage factors (8) are close to 1. This situation changes for the generalized shrinkage (12). The estimate of the generalized shrinkage parameter $\rho$ is $\hat{\rho}=0.038$. The mean values of shrinkage factors and of estimated prediction errors based on one-curve-leave-out cross-validation are shown in Table 2 . We find that gains of $4 \%$ are realized in this example over Gaussian shrinkage by using generalized shrinkage.

From the estimates of the first FPC scores, $\hat{\xi}_{i 1}$, we find that subjects \#3 and \#11 have the first and second largest FPC scores with regard to the first eigenfunction, corresponding to the first and second highest peaks. Subjects \#7, \#8, \#9 and \#10 have large negative scores corresponding to low peaks. The predicted trajectories for individual subjects are shown in Figure 5, based on generalized shrinkage estimates. Since there are about 40 measurements for each subject, the time points are dense after transformation, so that the proposed method is quite feasible. One can see that the predictions are reasonably close to the observations except for minor deviations near the peaks. To illustrate the effectiveness of the predictions, we also show predictions after transforming the time back to the original scale. The observations and predictions for the 12 subjects, excluding Subject \#2, are shown in Figure 6 for the first three days. The agreement between observed and fitted values indicates that the proposed method reasonably explains the dynamic behavior of the plasma ${ }^{14} \mathrm{C}$-folate specific activity responses to a small oral dose of 
${ }^{14} \mathrm{C}$-folic acid.

\section{Concluding Remarks}

In this paper we propose Gaussian and generalized shrinkage estimates for the FPC scores. The shrinkage estimates are simple to obtain and improve upon the customary integral approximation, both from a theoretical as well as practical viewpoint. For the folate data, generalized shrinkage leads to improved estimates of the FPC scores as manifested by improved cross-validation prediction errors. We also introduced scatter-plot smoothing methods that are specially adapted to the presence of additional measurement errors. By fitting local quadratic components along the direction perpendicular to the diagonal, we improve estimation in the neighborhood of and at the diagonal of the covariance surface. A square-root logarithm time transformation is shown to address the increasing time lags and sparsity of measurements in the right tail.

The proposed nonparametric method combining data-driven flexible features and shrinkage estimates seems well suitable for the description of samples of biological trajectories. Integrated squared residual error is shown to be a useful criterion for diagnostics and the detection of outliers. One such outlier was identified by this criterion and was confirmed to belong to a biologically different population.

\section{ACKNOWLEDGMENTS}

The Human Subjects Review Committees at the University of California Davis and at the Lawrence Livermore National Laboratory approved the study. This research was supported in part by NIH Grant R01 DK45939 and by NSF Grants DMS 99-71602 and DMS 02-04869. We wish to thank two reviewers and an associate editor for helpful comments.

\section{REFERENCES}

Berkey, C. S., and Kent, R. L., Jr. (1983). Longitudinal Principal Components and Non-Linear Regression Models of Early Childhood Growth. Annals of Human Biology 10, 523-536.

Besse, P., and Ramsay, J.O. (1986). Principal Components Analysis of Sampled Functions. Psychometrika 51, 285-311.

Capra, W.B., and Müller H.G. (1997). An Accelerated-Time Model for Response Curves. Journal 
of the American Statistical Association 92, 72-83.

Castro, P. E., Lawton, W. H., and Sylvestre, E. A. (1986). Principal Modes of Variation for Processes With Continuous Sample Curves. Technometrics 28, 329-337.

Clifford, A.J., Arjomand, A., Dueker, S.R., Schneider, P.D., Buchholz, B.A., and Vogel, J.S. (1998). The Dynamics of Folate Acid Metabolism in an Adult Given a Small Tracer Dose of ${ }^{14}$ C-Folic Acid. Advances in Experimental Medicine and Biology 445, 239-251.

Courant, R., and Hilbert, D. (1953). Methods of Mathematical Physics. Interscience, New York.

Fan, J., and Gijbels, I. (1996). Local Polynomial Modelling and Its Application. Chapman and Hall, London.

Gasser, T., Müller, H.G., Köhler, W., Molinari, L., and Prader, A. (1984). Nonparametric Regression Analysis of Growth Curves. Annals of Statistics 12, 210-229.

Lin, X., and Carroll, R. J. (2000). Nonparametric Function Estimation for Clustered Data When the Predictor is Measured Without/With Error. Journal of the American Statistical Association 95, 520-534.

Müller, H.G., and Prewitt, K. (1993). Multiparameter Bandwidth Processes and Adaptive Surface Smoothing. Journal of Multivariate Analysis 47 1-21.

Ramsay, J., and Silverman, B. (1997). Functional Data Analysis, Springer, New York .

Rice, J., and Silverman, B. (1991). Estimating the Mean and Covariance Structure Nonparametrically When the Data are Curves. Journal of the Royal Statistical Society 53, 233-243.

Rice, J., and Wu, C. (2000). Nonparametric Mixed Effects Models for Unequally Sampled Noisy Curves. Biometrics 57, 253-259.

Silverman, B. (1996). Smoothed Functional Principal Components Analysis by Choice of Norm. Annals of Statistics 24, 1-24.

Staniswalis, J. G., and Lee, J. J. (1998). Nonparametric Regression Analysis of Longitudinal Data. Journal of the American Statistical Association 93, 1403-1418. 
Table 1: Results of 100 Monte Carlo runs with $N=10,20,50$ trajectories per sample. Shown are averages of estimates $\rho=\hat{\sigma}^{2}$ (4) for Gaussian (8) and of estimates $\rho=\hat{\rho}$ (11) for generalized (12) shrinkage, cross-validation scores CV (11), squared prediction errors SPE (13) and average squared errors (ASE) for the two functional principal component scores $\xi_{1}$ and $\xi_{2}$.

\begin{tabular}{|c|c|c|c|c|c|c|c|}
\hline & Model & \multicolumn{3}{|c|}{ Normal } & \multicolumn{3}{|c|}{ Mixture } \\
\hline \multirow[t]{2}{*}{$\begin{array}{c}\text { Sample } \\
\text { Size }\end{array}$} & $\begin{array}{c}\text { Shrinkage } \\
\text { Type }\end{array}$ & None & $\begin{array}{l}\text { Gaussian } \\
\left(\rho=\hat{\sigma}^{2}\right)\end{array}$ & $\begin{array}{l}\text { Generalized } \\
(\rho=\hat{\rho})\end{array}$ & None & $\begin{array}{l}\text { Gaussian } \\
\left(\rho=\hat{\sigma}^{2}\right)\end{array}$ & $\begin{array}{l}\text { Generalized } \\
(\rho=\hat{\rho})\end{array}$ \\
\hline & True $\sigma^{2}$ & - & .250 & - & - & .250 & - \\
\hline \multirow{5}{*}{$N=10$} & Ave. $\rho$ & - & .273 & .945 & - & .271 & 1.34 \\
\hline & Ave. CV & 1.35 & 1.24 & 1.19 & 1.32 & 1.26 & 1.16 \\
\hline & Ave. SPE & .971 & .909 & .873 & .964 & .908 & .862 \\
\hline & $\operatorname{ASE}\left(\xi_{1}\right)$ & .386 & .361 & .354 & .371 & .353 & .338 \\
\hline & $\operatorname{ASE}\left(\xi_{2}\right)$ & .442 & .414 & .398 & .453 & .438 & .409 \\
\hline \multirow{5}{*}{$N=20$} & Ave. $\rho$ & - & .261 & .881 & - & .267 & 1.15 \\
\hline & Ave. CV & 1.14 & 1.06 & 1.03 & 1.12 & 1.04 & .985 \\
\hline & Ave. SPE & .866 & .814 & .793 & .910 & .876 & .831 \\
\hline & $\operatorname{ASE}\left(\xi_{1}\right)$ & .352 & .334 & .327 & .336 & .328 & .315 \\
\hline & $\operatorname{ASE}\left(\xi_{2}\right)$ & .417 & .396 & .389 & .425 & .409 & .384 \\
\hline \multirow{5}{*}{$N=50$} & Ave. $\rho$ & - & .257 & .421 & - & .259 & .578 \\
\hline & Ave. CV & .961 & .893 & .887 & .948 & .887 & .875 \\
\hline & Ave. SPE & .812 & .767 & .762 & .830 & .781 & .774 \\
\hline & $\operatorname{ASE}\left(\xi_{1}\right)$ & .343 & .329 & .319 & .331 & .320 & .308 \\
\hline & $\operatorname{ASE}\left(\xi_{2}\right)$ & .406 & .387 & .371 & .408 & .398 & .381 \\
\hline
\end{tabular}


Table 2: Shrinkage estimation of functional principal component scores for folate data. Average shrinkage parameter (ASP) is $\rho=\hat{\sigma}^{2}$ (4) for Gaussian shrinkage and $\rho=\hat{\rho}$ (11) for generalized shrinkage. Average shrinkage factor for the $k$ th functional principal component score is $\operatorname{ASF}\left(\xi_{k}\right)=$ $\frac{1}{N} \sum_{i=1}^{N}\left(\hat{\xi}_{i k} / \hat{\eta}_{i k}\right), k=1,2,3$, using (8) for Gaussian and (12) for generalized shrinkage, and CV denotes the observed minimum of cross-validation score (6).

\begin{tabular}{c|lll}
\hline \hline Shrinkage Type & None & Gaussian & Generalized \\
\hline $\mathrm{ASP}$ & - & 0.0037 & 0.038 \\
\hline $\operatorname{ASPF}\left(\xi_{1}\right)$ & 1 & .999 & .987 \\
\hline $\operatorname{ASPF}\left(\xi_{2}\right)$ & 1 & .987 & .886 \\
\hline $\operatorname{ASPF}\left(\xi_{3}\right)$ & 1 & .972 & .771 \\
\hline $\mathrm{CV}$ & .355 & .354 & .342 \\
\hline
\end{tabular}
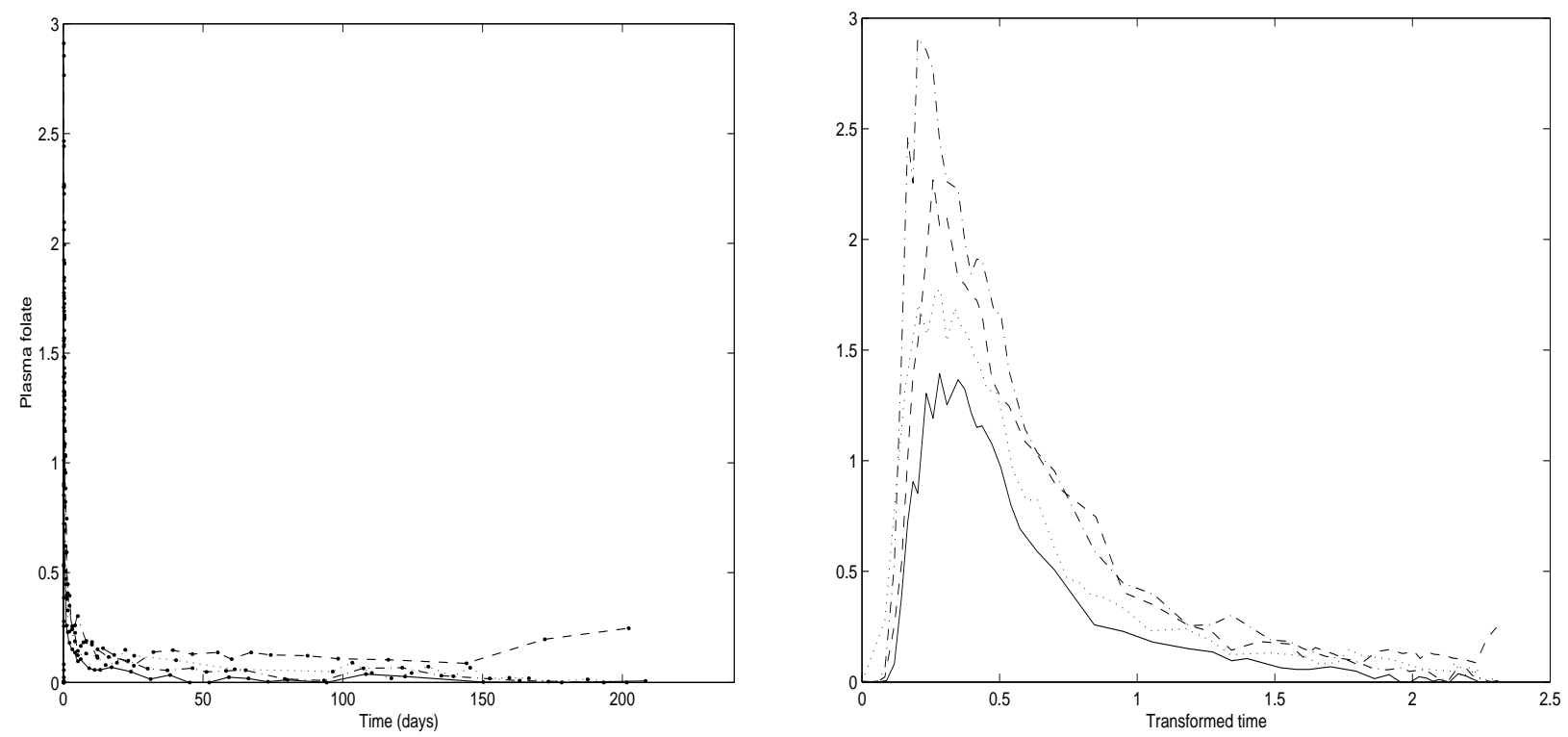

Figure 1: Left: Observed individual trajectories for the first 4 subjects, \#1(solid), \#2(dashed), \#3(dashdot), \#4(dotted), in the original time scale. Right: The same four individual trajectories shown in the transformed time scale. 


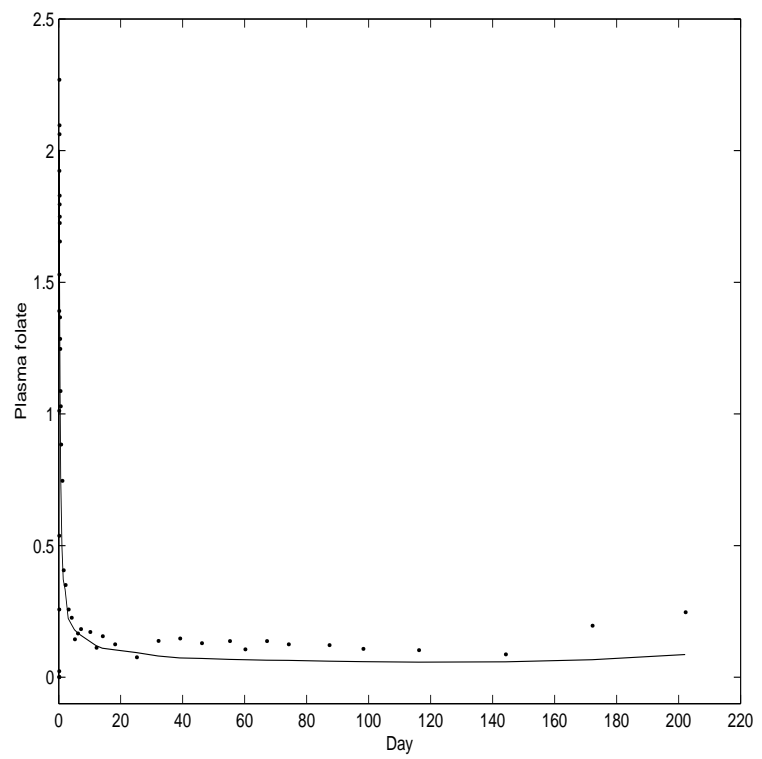

Figure 2: Observed values (dots) and predictions (solid) for subject \#2 in original time scale.
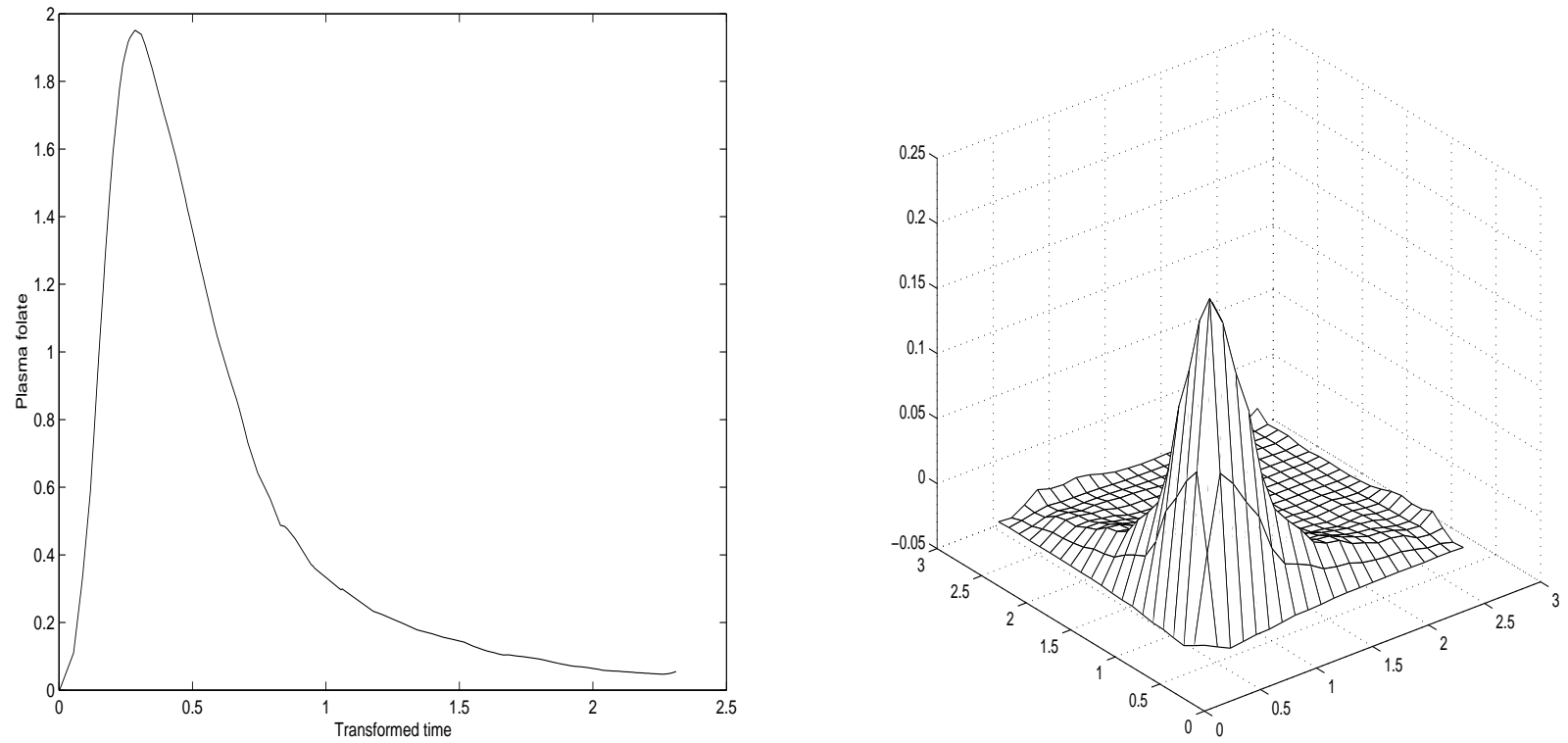

Figure 3: Left: Smooth estimate of the mean function (transformed time scale). Right: Smooth estimate of the covariance surface (transformed time scale). 


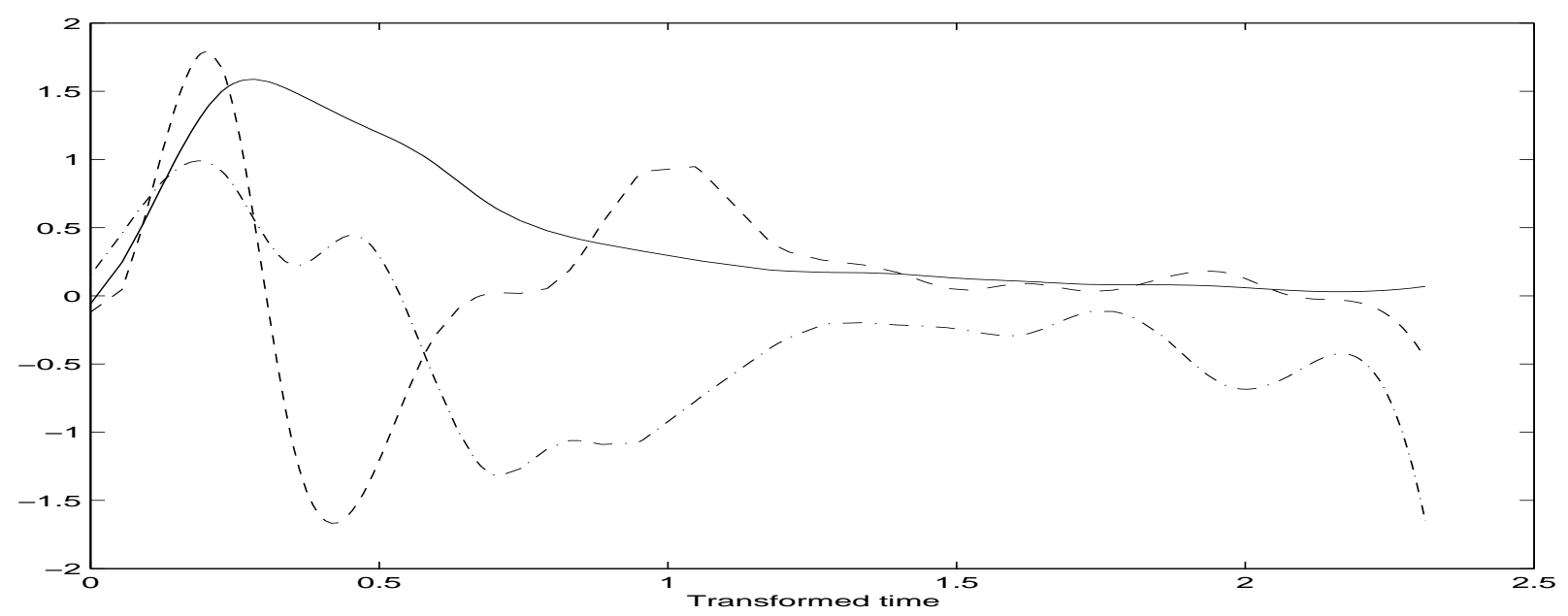

Figure 4: The first three eigenfunctions in the transformed time scale, first (solid), second (dashed), and third eigenfunction (dash-dot).
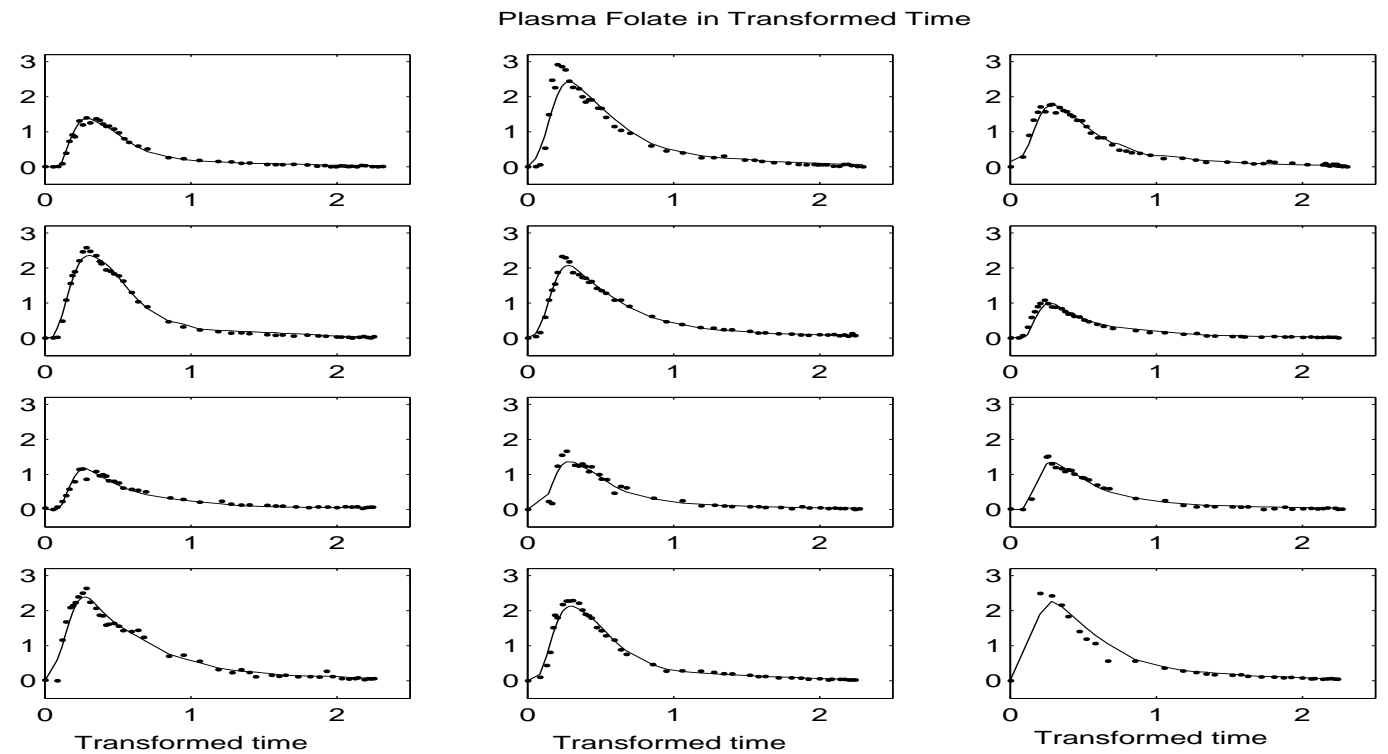

Figure 5: Observed (dots) and predicted (solid curves) folate values for the 12 subjects (excluding Subject \#2), shown in transformed time scale. 

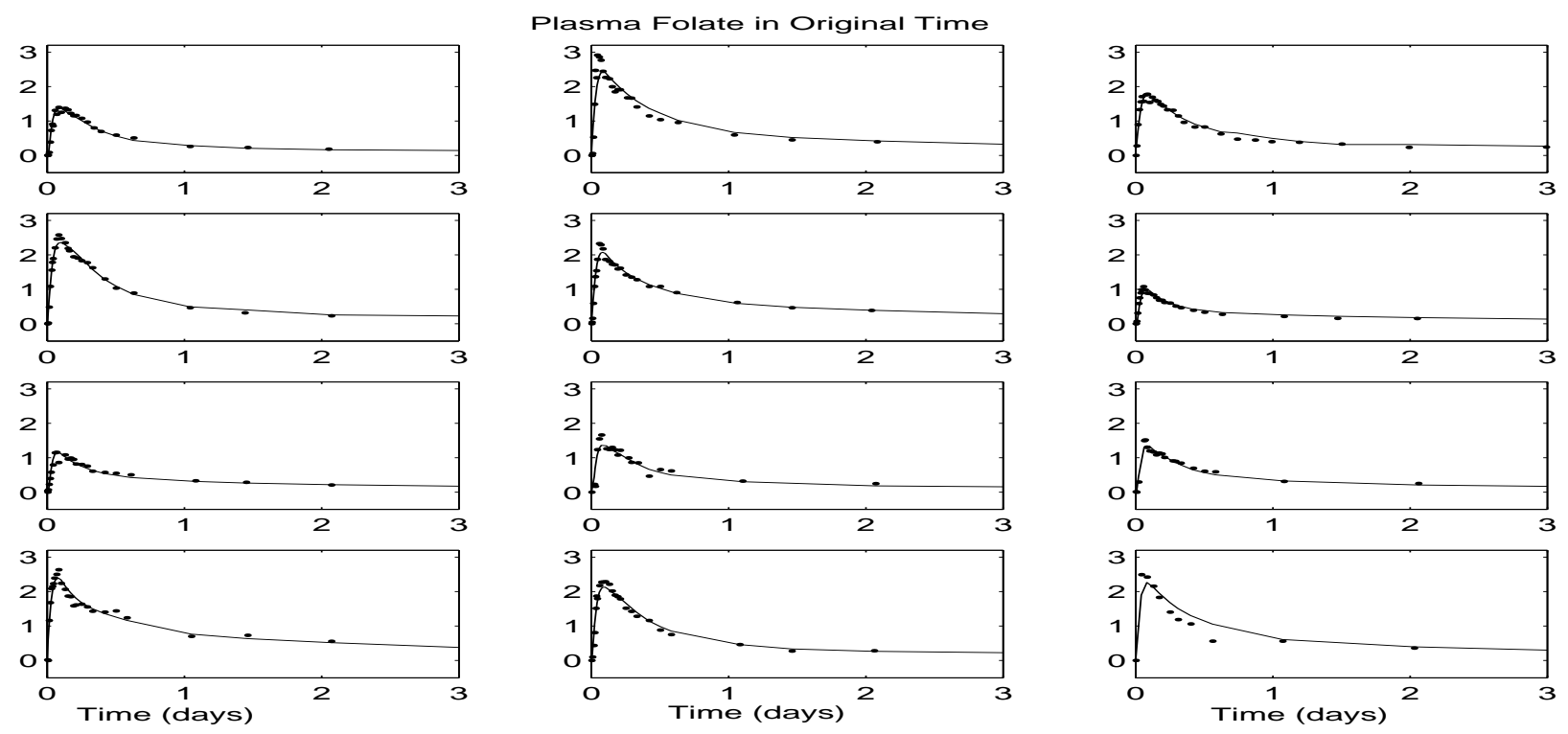

Figure 6: Same as Figure 5 for the first three days, shown in the original time scale. 